\title{
Statistical analysis of the mechanical properties of composite materials
}

\author{
E. Barbero, J. Fernández-Sáez, C. Navarro* \\ Department of Mechanical Engineering, Carlos III University of Madrid, Avda. de la Universidad, 30, 28911-Leganés, Madrid, Spain
}

\begin{abstract}
The Weibull statistic is currently used in designing mechanical components made of composite materials. This work presents useful formulae to describe the behaviour of the Weibull modulus estimator, which in turn may be described by means of a three parameter Weibull distribution. Expressions for the parameters of this latter distribution, dependent on the sample size, are also given in the paper, so, the percentage points, published until now in tabular form, may be directly calculated. Empirical expressions are derived for determining the $A$ basis and the $B$ basis material properties as a function of the sample size.
\end{abstract}

Keywords: C. Statistical methods; B. Mechanical properties; B. Strength; Composite materials

\section{Introduction}

Unlike most traditional structural materials, whose mechanical behaviour is assumed to be homogeneous and isotropic, mechanical properties of composite materials exhibit intrinsic statistical dependence. In particular, their strength properties are usually scattered due to their inhomogeneity and anisotropic characteristics and to the brittleness of the matrices and fibres. So, careful statistical analysis is indispensable for the understanding of the mechanical characterisation of these materials.

The Weibull statistic [1] has been widely used in the recent years to describe the statistical behaviour of the strength properties of many materials, such as advanced ceramics [2], metallic matrix composites [3], ceramic matrix composites [4], and polymeric matrix composites [5]. The Weibull distribution also describes the fatigue behaviour of materials and the scatter of the fracture toughness of steels in the ductile-brittle transition region, where failure occurs by cleavage [6,7].

The two-parameter Weibull distribution function is given by:

$F(\sigma) \quad 1-\exp \left[-\left(\frac{\sigma}{\sigma_{0}}\right)^{m}\right]$

where $F$ is the probability of rupture of the material under uniaxial tensile stress $\sigma, m$ is the shape parameter or
Weibull modulus, and $\sigma_{0}$ is the scale parameter of the distribution. Weibull modulus, $m$, is related to the scatter of the data: the higher the $m$ the lower the dispersion of fracture stress. It becomes the most important parameter of the distribution. The scale parameter is closely related to the mean fracture stress.

Since the evaluation of the parameters of Weibull distribution is made from a finite number of tests, the estimators of their true values have a statistical character, and thus the uncertainty of the estimation must be known. In this work a three-parameter Weibull distribution is also proposed for the estimator of the Weibull modulus. Formulae for the three parameters defining such a distribution, dependent only on the sample size, are given below.

To design structural and mechanical components, the determination of the $\sigma$-values, corresponding to a predefined failure probability, is of great interest to the engineer. These values coincide with their percentiles of the distribution. In particular, the values corresponding to the percentiles of 90 and $99 \%$, estimated with a confidence level of 95\%, are known [8] as the A basis and B basis material property, respectively. These values are obtained from the estimations of $m, \sigma_{0}$, and in terms of two parameters, $P_{\mathrm{A}}$ and $P_{\mathrm{B}}$, which depend only on the sample size and on the estimation method used, and are usually obtained from tables [8]. In this paper, to simplify the calculus of these parameters, fitted equations are developed for two estimation methods: maximum-likelihood and weighted regression. 


\section{Theoretical background}

Several procedures are available for the determination of the Weibull distribution parameters. From the maximum likelihood method, the estimators of the Weibull parameters, $\hat{m}$ and $\hat{\sigma}_{0}$, should satisfy the following equations:

$\sum_{i=1}^{i=n} \ln \sigma_{i}-n\left(\frac{\sum_{i=1}^{i=n} \ln \sigma_{i}\left(\sigma_{i}\right)^{\hat{m}}}{\sum_{i=1}^{i=n}\left(\sigma_{i}\right)^{\hat{m}}}\right)+\frac{n}{\hat{m}} \quad 0$

and

$\hat{\sigma}_{0} \quad\left(\frac{1}{n} \times \sum_{i=1}^{i=n}\left(\sigma_{i}\right)^{\hat{m}}\right)^{1 / \hat{m}}$

Although Eq. (2) is non-linear, it has a unique positive solution [9], and may be solved by the Newton-Raphson iteration technique or by any other method.

Eq. (1) becomes a straight line if a double logarithmic transformation is made, i.e.

$\ln \left[\ln \left(\frac{1}{1-F(\sigma)}\right)\right] \quad m \ln \sigma-m \ln \sigma_{0}$

The $F$ values are assigned on the basis of the $i$ th position of a value among the $n$ ordered $\sigma$-values forming the sample. Thus the general linear regression method may be used to obtain $\hat{m}$ and $\hat{\sigma}_{0}$.

Several estimators of $F$ can be considered

$$
\begin{array}{ll}
F_{i} & \frac{i-0.5}{n} \\
F_{i} & \frac{i-0.3}{(n+0.4)} \\
F_{i} & \frac{i}{(n+1)}
\end{array}
$$

Different authors $[10,11]$ have pointed out the convenience of using a weight function, $W_{i}$, in performing the linear regression. Bergman [10] proposed the weight factor given by

$$
W_{i} \quad\left[\left(1-F_{i}\right) \times \ln \left(1-F_{i}\right)\right]^{2}
$$

and Faucher and Tyson [11] suggested the following:

$$
W_{i} \quad 3.3 F_{i}-27.5\left[1-\left(1-F_{i}\right)^{0.025}\right]
$$

Using the general linear regression method, from the $n$ pairs of values $(\sigma-F)$, the estimators of the Weibull parameters can be calculated as

$\hat{m} \frac{\left(\sum W_{i}\right)\left(\sum W_{i} z_{i} y_{i}\right)-\left(\sum W_{i} z_{i}\right)\left(\sum W_{i} y_{i}\right)}{\left(\sum W_{i}\right)\left(\sum W_{i} z_{i}^{2}\right)-\left(\sum W_{i} z_{i}\right)^{2}}$ and

$\hat{\sigma}_{0} \exp \left(-\frac{a}{\hat{m}}\right)$

where

$z_{i} \quad \ln \sigma_{i}$

$y_{i} \quad \ln \left[\ln \left(\frac{1}{1-F_{i}}\right)\right]$

$a \frac{\sum W_{i} y_{i}-\hat{m} \sum W_{i} z_{i}}{\sum W_{i}}$

Finally, applying the moments method, in which the sample moments are equated to those of the distribution, the estimators of $m$ and $\sigma_{0}$ are the solutions of

$$
\frac{\bar{\sigma}}{S} \frac{\Gamma\left(1+\frac{1}{\hat{m}}\right)}{\left[\Gamma\left(1+\frac{2}{\hat{m}}\right)-\Gamma^{2}\left(1+\frac{1}{\hat{m}}\right)\right]^{1 / 2}}
$$

and

$$
\hat{\sigma}_{0} \frac{\hat{\sigma}}{\Gamma\left(1+\frac{1}{\hat{m}}\right)}
$$

where $\bar{\sigma}$ and $S$ are, respectively, the mean and the standard deviation of the experimental data, and $\Gamma$ is the Gamma function. Eq. (13) may be solved using the Newton-Raphson procedure.

Thoman et al. [12] showed that if the maximum likelihood method were used, the variables $\hat{m} / m$ and $\hat{m} \ln \left(\hat{\sigma}_{0} / \sigma_{0}\right)$ are distributed independently of the true values of $m$ and $\sigma_{0}$, and they have the same distribution as $\hat{m}_{11}$ and $\hat{m}_{11} \ln \left(\hat{\sigma}_{0}\right)_{11}$, respectively, which correspond to $m \quad 1$ and $\sigma_{0} \quad 1$. The percentage points of these distributions were calculated by Thoman et al. [12] from Monte Carlo simulations.

For a two-parameter Weibull distribution, the A-basis, $\sigma_{\mathrm{A}}$, and B-basis material properties, $\sigma_{\mathrm{B}}$, might be obtained [8] as

$$
\begin{array}{ll}
\sigma_{\mathrm{A}} & \hat{\sigma}_{0}\left(\ln \left(\frac{1}{P_{\mathrm{A}}}\right)\right)^{1 / \hat{m}} \\
\sigma_{\mathrm{B}} & \hat{\sigma}_{0}\left(\ln \left(\frac{1}{P_{\mathrm{B}}}\right)\right)^{1 / \hat{m}}
\end{array}
$$

Usually the values of the parameters $P_{\mathrm{A}}$ and $P_{\mathrm{B}}$ are calculated by means of the maximum-likelihood method and, so far, their values have been presented in a tabular form [8]. Herein the values of $P_{\mathrm{A}}$ and $P_{\mathrm{B}}$ may be computed as

$$
\begin{aligned}
& P_{\mathrm{A}} \quad(1-0.01)^{\exp \left(-C_{001}\right)} \\
& P_{\mathrm{B}} \quad(1-0.1)^{\exp \left(-C_{01}\right)}
\end{aligned}
$$

where the constants $C_{0.01}$ and $C_{0.1}$ are, respectively, the 95 


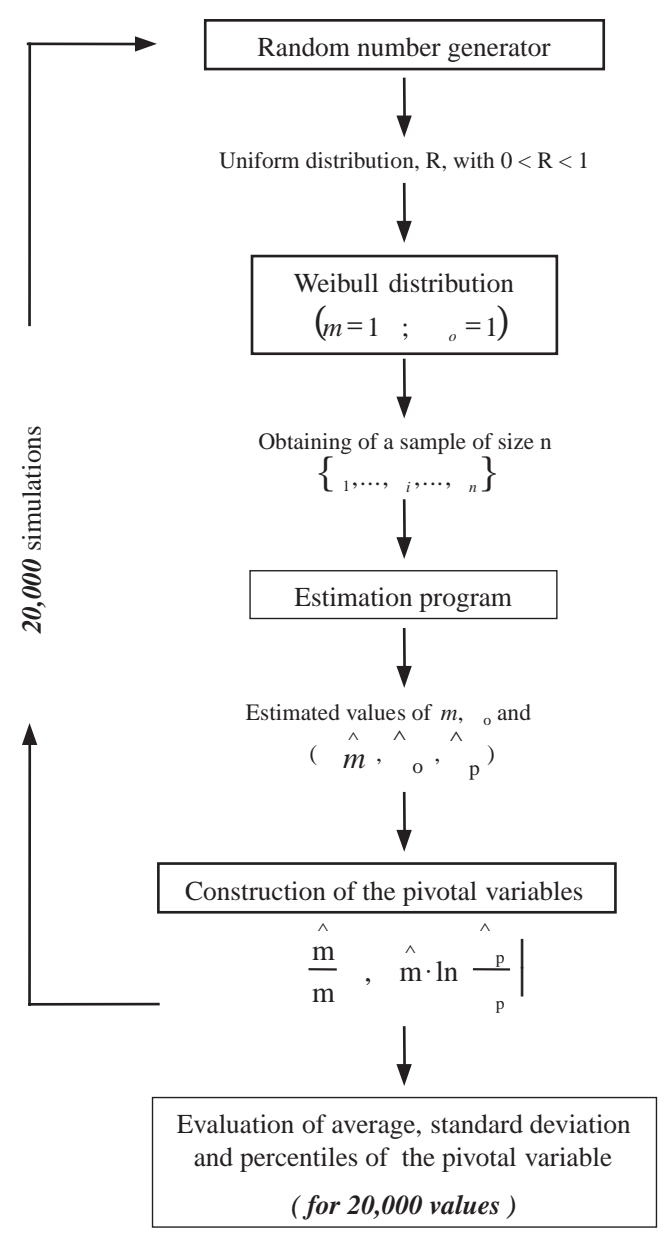

Fig. 1. Flow chart of the simulation procedure.

percentage points of the pivotal variables $\hat{m} \ln \left(\hat{\sigma}_{0.01} / \sigma_{0.01}\right)$ and $\hat{m} \ln \left(\hat{\sigma}_{0.1} / \sigma_{0.1}\right)$, i.e. should verify

$$
\begin{aligned}
& \operatorname{Pr}\left[\hat{m} \ln \left(\frac{\hat{\sigma}_{0.01}}{\sigma_{0.01}}\right) \leq C_{0.01}\right] \quad 0.95 \\
& \operatorname{Pr}\left[\hat{m} \ln \left(\frac{\hat{\sigma}_{0.1}}{\sigma_{0.1}}\right) \leq C_{0.1}\right] \quad 0.95
\end{aligned}
$$

Fernández-Sáez et al. [13] showed that the variable $\hat{m} \ln \left(\hat{\sigma}_{p} / \sigma_{p}\right)$ is distributed independently of the true percentile value and, Barbero et al. [14] have proved that, in order to estimate the percentiles of the two-parameter Weibull distribution, the best method is the weighted regression one, using Eq. (5c) to estimate $F_{i}$, and Eq. (7) to calculate $W_{i}$, if sample size is above 7, or Eq. (6) otherwise.

Table 1

Average and standard deviation of variable $\hat{m} / m$, according to Eq. (20)

\begin{tabular}{lrlll}
\hline & \multicolumn{2}{l}{ Parameter } & & \\
\cline { 2 - 5 } & $A$ & $B$ & \multicolumn{1}{l}{$C$} & \multicolumn{1}{l}{ D } \\
\hline Average & 0.9807 & 1.7001 & 2.5873 & 1.0408 \\
Standard deviation & -0.1357 & 0.5297 & 0.7303 & 0.3087 \\
\hline
\end{tabular}

\section{Numerical simulation}

To obtain the statistical distribution of the pivotal variables $\hat{m} / m$ and $\hat{m} \ln \left(\hat{\sigma}_{p} / \sigma_{p}\right)$, a simulation procedure, based on the Monte Carlo method, has been used. In this procedure (see Fig. 1 for better understanding) a set of $n$ values was generated as

$\sigma_{i} \quad \sigma_{0} \ln \left(\frac{1}{R}\right)^{1 / m}$

In this work we select $m \quad 1$ and $\sigma_{0} \quad 1$. Note that, as stated above, the analysis is independent of the true values [12].

$R$ is a random variable with uniform distribution in the $[0,1]$ interval. From each sample so obtained, $\left\{\sigma_{1}, \sigma_{2}, \sigma_{3}, \ldots, \sigma_{n}\right\}$, estimations of the Weibull parameters, $\hat{m}$ and $\hat{\sigma}_{0}$, and the $p$-percentile $\hat{\sigma}_{p}$, were obtained. From these estimations, the variables $\hat{m} / m$ and $\hat{m} \ln \left(\hat{\sigma}_{p} / \sigma_{p}\right)$ could also be built. Repeated application of this procedure provides a statistical distribution for the two latter variables. In this work we have computed 20,000 values for $\hat{m} / \mathrm{m}$ and $\hat{m} \ln \left(\hat{\sigma}_{p} / \sigma_{p}\right)$ for each sample size, which, in turn was progressively increased from 5 up to 120 .

The values of $C_{0.01}$ and $C_{0.1}$, defined by Eqs. (18a) and (18b), were also computed, and from these, the values of $P_{\mathrm{A}}$ and $P_{\mathrm{B}}$ were obtained using Eqs. (17a) and (17b), respectively.

For the estimation of $\hat{m} / m$, the maximum-likelihood method (method 1) was utilised, while the authors have used three estimation methods for the calculation of $P_{\mathrm{A}}$ and $P_{\mathrm{B}}$ : maximum-likelihood (method 1) and weighted regression, with two different weighted functions: Eq. (6) (method 2), and Eq. (7) (method 3).

\section{Parameter fitting}

From the 20,000 values of the pivotal variable $\hat{m} / m$, its average value, $(\hat{m} / \mathrm{m})_{\text {ave }}$ was calculated. In order to fit this value to the sample size, the following four-parameter function is proposed:

$\left(\frac{\hat{m}}{m}\right)_{\text {ave }} A+B\left(\frac{1}{\ln (D n)}\right)^{C}$

where $A, B, C$, and $D$ are the fit parameters given in Table 1 , and $n$ is the sample size. As shown in Fig. 2, the fit seems to be very good, with a maximum error of $0.23 \%$. If parameter $D$ were taken as unity, the increment of the maximum error would become negligible and thus three parameters would suffice. The average value of the pivotal variable approaches unity (the estimator approach to the true value) when sample size $n$ increases, which agrees with the Khalili et al. [15] results.

The standard deviation of the variable $\hat{m} / m$ was computed as a function of the sample size. Its value decreases to zero as the sample size increases, as Khalili et al. [15] observed. 


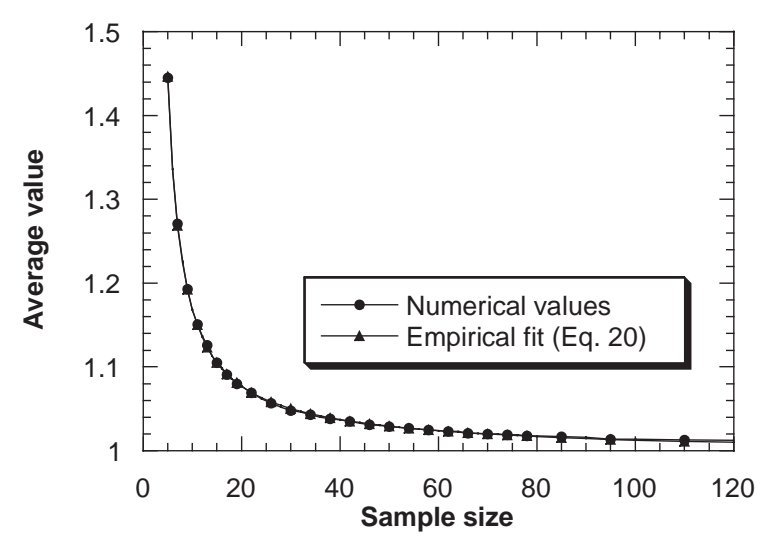

Fig. 2. Variation of the average of the variable $\hat{m} / m$ as a function of the sample size, $n$. Empirical fit according to Eq. (20).

To fit the standard deviation we used the same type of function as before for the average value. Fig. 3 shows the dependence of the standard deviation on the sample size. The fit parameters are given in Table 1 and the maximum error was $2.07 \%$.

Percentage points of the pivotal variable were also calculated numerically. These results, published by Thoman et al. [12] in tabular form, showed that the variable $\hat{m} / m$ is normal asymptotically with an average value of 1 and standard deviation $\sqrt{0.608 / n}$, which means that the percentiles of the variable could be calculated from those of a normal distribution. One improvement to this approach would be to consider that the variable $\hat{\mathrm{m}} / \mathrm{m}$ is normally distributed with the average and the standard deviation given by Eq. (20) using the parameter obtained from Table 1.

In this paper a three-parameter Weibull distribution is proposed for the variable $\hat{m} / m$, i.e.

$F \quad 1-\exp \left[-\left(\frac{(\hat{m} / m)-P_{1}}{P_{2}}\right)^{P_{3}}\right]$

where $P_{1}, P_{2}$, and $P_{3}$ are, respectively, the position, scale and form parameters, that may be fitted as a function of the

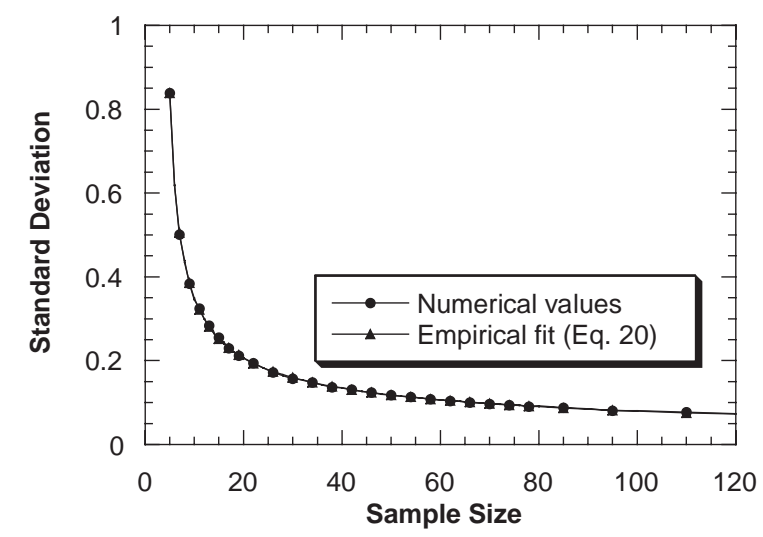

Fig. 3. Variation of the standard deviation of the variable $\hat{m} / \mathrm{m}$ as a function of sample size, $n$. Empirical fit according to Eq. (20).
Table 2

Three-parameter Weibull distribution for the variable $\hat{m} / m$, according to Eqs. (21) and (22)

\begin{tabular}{|c|c|c|c|}
\hline & \multicolumn{3}{|l|}{ Parameter } \\
\hline & $m_{1}$ & $m_{2}$ & $m_{3}$ \\
\hline$P_{1}$ & 0.5268 & 0.0591 & 1.0572 \\
\hline$P_{2}$ & -0.2109 & 1.5299 & -0.8318 \\
\hline$P_{3}$ & 0.2297 & 0.7140 & 0.8027 \\
\hline
\end{tabular}

sample size, $n$, by

$$
P_{i} \quad m_{i 1}+m_{i 2}(\ln n)^{m_{i 3}} ; \quad i \quad 1,2,3
$$

the empirical parameters $m_{i 1}, m_{i 2}$, and $m_{i 3}$ being those shown in Table 2.

This last approach seems to be better than those cited above for the calculation of the percentiles, as shown in Fig. 4, which gives the variation of the error with the sample size for the 0.985 percentile of the variable $\hat{m} / m$ using different methods: the three-parameter Weibull distribution; normal distribution of average 1 and standard deviation $\sqrt{0.608 / n}$; and finally normal distribution with the average and standard deviation values calculated from Eq. (20).

Also, the values of $P_{\mathrm{A}}$ and $P_{\mathrm{B}}$, obtained using Eqs. (17a) and $(17 \mathrm{~b})$ from the numerical simulation results have been fitted by means of the following equation:

$$
P_{\mathrm{A}}\left(\text { or } P_{\mathrm{B}}\right) \quad M_{1}+M_{2} \exp \left(n^{-M_{3}}\right)
$$

The parameters $M_{1}, M_{2}$, and $M_{3}$ are shown in Tables 3 and 4 . Fig. 5 shows a comparison of the numerical values of $P_{\mathrm{B}}$ with the corresponding ones obtained from Eq. (23). The fits for both $P_{\mathrm{A}}$ and $P_{\mathrm{B}}$ are very good, with errors below $0.3 \%$ in all the cases. Once $P_{\mathrm{A}}$ and $P_{\mathrm{B}}$ values are known, the A-basis and B-basis material properties may be obtained from Eqs. (15) and (16).

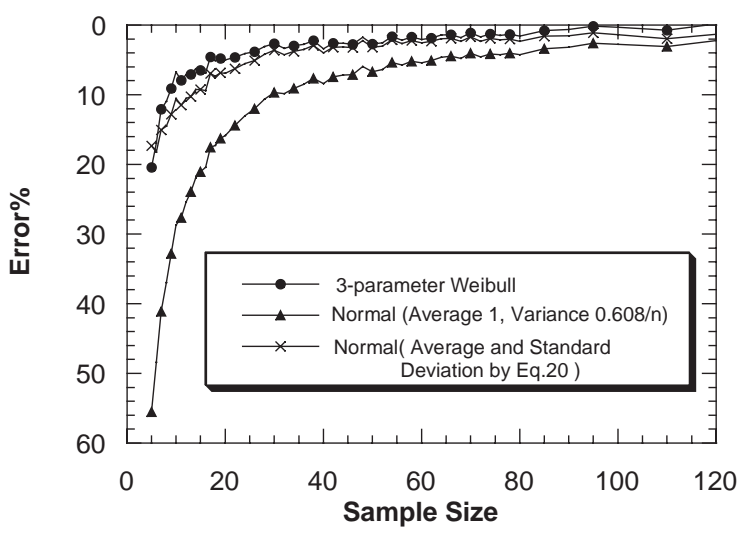

Fig. 4. Error of estimation of the 0.985 percentile of the variable $\hat{m} / m$ for different methods. Curve 1: three-parameter Weibull distribution. Curve 2: normal of average 1 and variance $0.608 / n$. Curve 3 : normal of average and variance calculated using Eq. (20). 
Table 3

Estimation of $P_{\mathrm{A}}$ as a function of sample size, according to Eq. (23)

\begin{tabular}{llllll}
\hline Method & $m_{1}$ & $m_{2}$ & $m_{3}$ & $R$ & Maximum error $(\%)$ \\
\hline 1 & 0.2758 & 0.2685 & 0.0030 & 0.9991 & 0.22 \\
2 & 0.4777 & 0.1928 & 0.0028 & 0.9981 & 0.01 \\
3 & 0.9766 & 0.0103 & 0.1250 & 0.9992 & 0.03
\end{tabular}

Table 4

Estimation of $P_{\mathrm{B}}$ as a function of sample size, according to Eq. (23)

\begin{tabular}{lrllll}
\hline Method & \multicolumn{1}{l}{$m_{1}$} & $m_{2}$ & $m_{3}$ & $R$ & Maximum error (\%) \\
\hline 1 & -1.3102 & 0.8651 & 0.0106 & 0.9980 & 0.30 \\
2 & 0.8132 & 0.0840 & 0.2227 & 0.9977 & 0.27 \\
3 & 0.8070 & 0.1003 & 0.3529 & 0.9914 & 0.17 \\
\hline
\end{tabular}

\section{Practical examples}

To illustrate the above methodology two examples are included in this paper, although it is worth noting that they can be applied in their integrity to any kind of composite material. In the first example, the estimation of the confidence intervals of the Weibull modulus for the static flexural strength of a CMC is considered, whereas in the second, the A-basis and B-basis values of the dynamic flexural strength of a CFRP are computed. In these examples, the loading rate and the sample size are different.

\subsection{Example 1: estimation of confidence intervals of Weibull modulus}

In the first example, the confidence intervals of the Weibull modulus (90\% and $99 \%$ confidence levels), for the static flexural strength of a ceramic matrix composite, are estimated.

The composite material was an alumina (CONDEA APA$0.5,99.97 \%$ of purity) matrix reinforced by $30 \%$ of zirconia particles (Tosoh $3 \mathrm{YB}$ ), processed by uniaxial pressing under $87 \mathrm{MPa}$ and pressureless sintering at $1650^{\circ} \mathrm{C}$ for $2 \mathrm{~h}$ [16].

Three-point bending tests of 15 prismatic specimens of

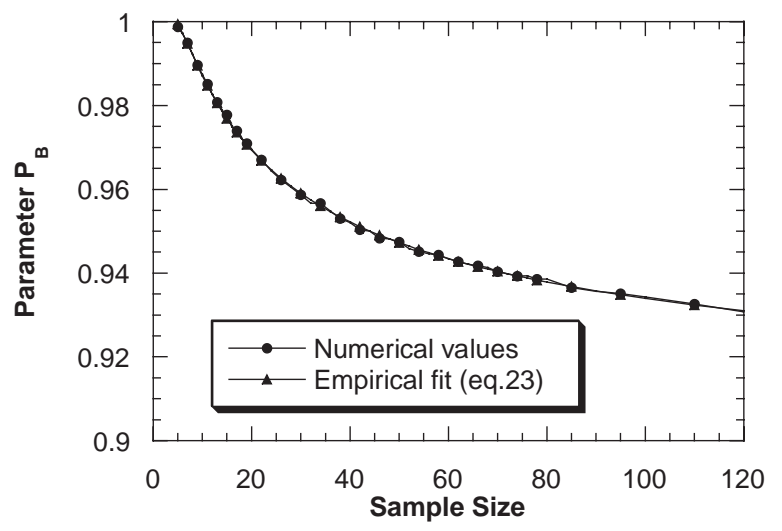

Fig. 5. Variation of the parameter $P_{\mathrm{B}}$ as a function of sample size, $n$. Empirical fit according to Eq. (23).
Table 5

Example 1: flexural strength (MPa) of a $30 \%$ zirconia alumina composite

\begin{tabular}{llcl}
\hline Sample & Strength (MPa) & Sample & Strength (MPa) \\
\hline 1 & 203 & 9 & 310 \\
2 & 225 & 10 & 325 \\
3 & 239 & 11 & 345 \\
4 & 265 & 12 & 360 \\
5 & 270 & 13 & 365 \\
6 & 275 & 14 & 400 \\
7 & 289 & 15 & 425 \\
8 & 295 & & \\
\hline
\end{tabular}

$10 \times 10 \times 55 \mathrm{~mm}^{3}$ were carried out using a universal testing machine (INSTRON 8016) with a $5 \mathrm{kN}$ load cell. The tests results (flexural strength) are shown in Table 5. From these values an estimation of the Weibull modulus and scale parameters were obtained by the maximum likelihood method [14], which gave

$\hat{m} \quad 5.398 ; \quad \hat{\sigma}_{0} \quad 331.5 \mathrm{MPa}$

Fig. 6 gives the experimental results and the fitted values using the Weibull distribution.

From the parameters $A, B, C$ and $D$ of Table 1 , the values of the average and standard deviation of the pivotal variable are calculated by Eq. (20) with $n \quad 15$ were

Average of $\left(\frac{\hat{m}}{m}\right) \quad 1.1050$

Standard deviation of $\left(\frac{\hat{m}}{m}\right) \quad 0.2521$

To find the percentage points of the estimator of the Weibull modulus, its distribution function, Eq. (21), may be used. The values of $P_{i}$ in this equation were computed from Eq. (22) and the parameters $m_{1}, m_{2}$ and $m_{3}$ from Table 2. For $n$ 15 the following values were obtained:

$\begin{array}{llllll}P_{1} & 0.6962 & P_{2} & 0.4571 & P_{3} & 1.8182\end{array}$

The percentiles of variable $\hat{m} / m$ to be used to calculate the confidence levels of the 99 and $90 \%$ are shown in Table 6. From such values the interval of the variable $m$, corresponding to the $99 \%$ confidence level, is

$2.934<m<7.487$

Table 6

Example 1: percentiles of variable $\hat{m} / m$, calculated according Eqs. (21) and (22)

\begin{tabular}{ll}
\hline Percentile & \multicolumn{1}{c}{ Value } \\
\hline $\begin{array}{ll}\text { Confidence level: } 99 \% \\
0.005\end{array}$ & 0.72103 \\
0.995 & 1.8398 \\
& \\
Confidence level: $90 \%$ & \\
0.050 & 0.78544 \\
0.950 & 1.5320 \\
\hline
\end{tabular}




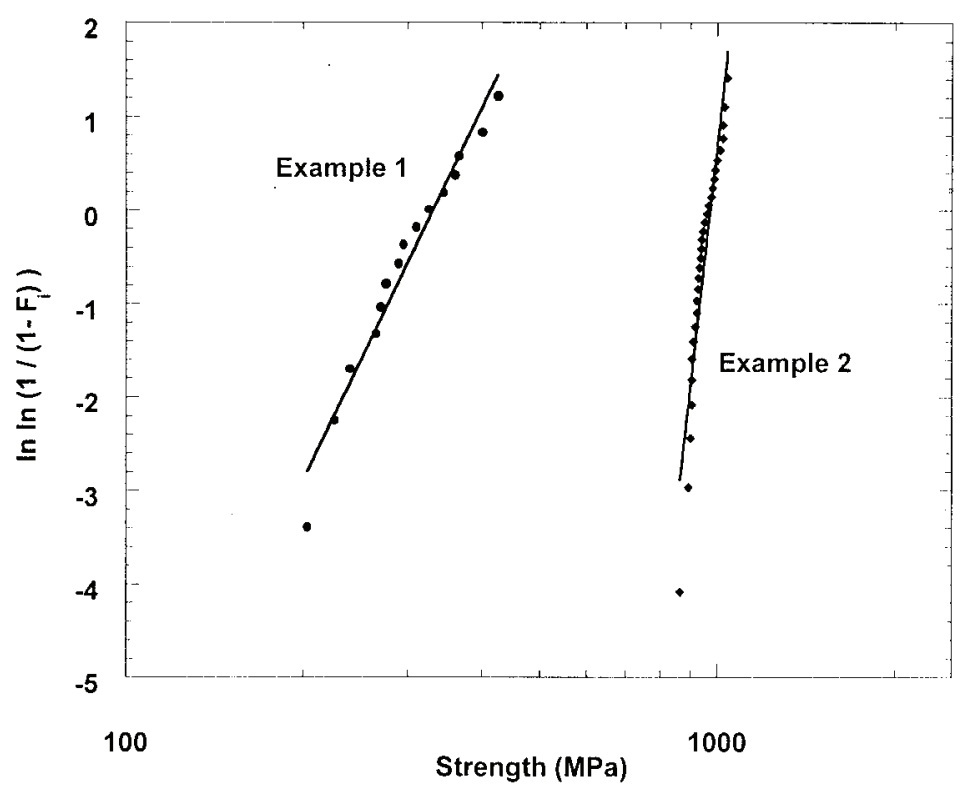

Fig. 6. Flexural strength distribution of an alumina 30\% zirconia composite (Example 1) and 0/90 carbon/epoxy laminate (Example 2).

and for the $90 \%$ confidence level

$3.523<m<6.873$

\subsection{Example 2: estimation of the A basis and B basis material properties}

In this example, the A-basis and B-basis material properties (dynamic flexural strength) of a polymeric matrix composite were calculated.

The composite material is a $2.4 \mathrm{~mm}$ thick carbon/epoxy, Hexcel AS4/350-61, [0/90] $]_{2 S}$ laminate, manufactured by SACESA.

Thirty dynamic three-point bending tests were performed using a CEAST Fractovis drop weight tester. The specimens were $2.4 \times 20 \times 105 \mathrm{~mm}^{3}$ beams. The drop weight was

Table 7

Example 2: flexural strength (MPa) of [0/90] carbon/epoxy composite

\begin{tabular}{llll}
\hline Sample & Strength $(\mathrm{MPa})$ & Sample & Strength $(\mathrm{MPa})$ \\
\hline 1 & 864 & 16 & 941 \\
2 & 891 & 17 & 945 \\
3 & 900 & 18 & 950 \\
4 & 904 & 19 & 959 \\
5 & 904 & 20 & 965 \\
6 & 904 & 21 & 975 \\
7 & 910 & 22 & 980 \\
8 & 915 & 23 & 986 \\
9 & 922 & 24 & 990 \\
10 & 922 & 25 & 998 \\
11 & 926 & 26 & 1010 \\
12 & 928 & 27 & 1022 \\
13 & 931 & 28 & 1022 \\
14 & 936 & 29 & 1027 \\
15 & 938 & 30 & 1040 \\
\hline
\end{tabular}

$3.608 \mathrm{~kg}$ and its velocity $4.08 \mathrm{~m} / \mathrm{s}$. The flexural strength results are given in Table 7 . For this example the estimations of the Weibull modulus and scale parameters were

$\hat{m} \quad 21.67 ; \quad \hat{\sigma}_{0} \quad 972.29 \mathrm{MPa}$

Fig. 6 gives the experimental results and the fitted values using the Weibull distribution.

From Eq. (23) and Tables 3 and 4, the parameters $P_{\mathrm{A}}$ and $P_{\mathrm{B}}$ are estimated for a sample size of 30 . The values obtained for the three methods cited in this article are shown in Table 8, which also gives the A-basis and Bbasis material properties.

\section{Summary}

This work presents useful formulae to analyse the variability of the mechanical properties of composite materials. The study is focused on the two-parameter Weibull distribution, currently used to describe statistically the strength properties of many kinds of materials. In order to obtain the percentage points of the estimator of the Weibull modulus, published until now in tabular form, a three-parameter Weibull distribution is proposed. Empirical expressions for these three parameters, dependent only on the sample size, are also given. The A-basis and B-basis material

Table 8

Example 2: estimation of A-basis and B-basis

\begin{tabular}{lllll}
\hline Method & $P_{\mathrm{A}}$ & $P_{\mathrm{B}}$ & $\sigma_{\mathrm{A}}(\mathrm{MPa})$ & $\sigma_{\mathrm{B}}(\mathrm{MPa})$ \\
\hline 1 & 0.9995 & 0.9728 & 694 & 834 \\
2 & 0.9976 & 0.9562 & 745 & 853 \\
3 & 0.9974 & 0.9519 & 749 & 857 \\
\hline
\end{tabular}


properties based on Weibull statistics can be derived from the estimations of the Weibull parameters and the values $P_{\mathrm{A}}$ and $P_{\mathrm{B}}$, which depend only on the sample size and the type of estimation method. Expressions for $P_{\mathrm{A}}$ and $P_{\mathrm{B}}$, estimated by the maximum likelihood, and two selected weightedregression methods, are given.

\section{Acknowledgements}

The authors are indebted to the Fundación Ramón Areces (Área de Materiales, IX Concurso Nacional) for the financial support of this research.

\section{References}

[1] Weibull W. A statistical function of wide applicability. Journal of Applied Mechanics 1951;18:293 7.

[2] Glaeser AM. The use of transient FGM interlayers for joining advanced ceramics. Composites Part B: Engineering 1997;28B:71 84.

[3] Fukui Y, Yamanaka N, Enokida Y. Bending strength of an $\mathrm{Al} \mathrm{Al}_{3} \mathrm{Ni}$ functionally graded material. Composites Part B: Engineering 1997;28B:37 43.

[4] Bloyce DM, Ham-Su R, Plucknett KP, Wilkinson DS. Tape casting of $\mathrm{SiC}$ platelet reinforced alumina. Advances in Ceramic Matrix Composites: Ceramic Transactions 1993;38:67 78.

[5] Lamela MJ, Fernández A, Reiter H, Viña J. Análisis experimental y estadístico del comportamiento a flexión dinámica de laminados de fibra de carbono y resina epoxi. Actas del I Congreso Nacional de Materiales Compuestos, (in Spanish) 1995. p. 33540.

[6] Evans AG. Statistical aspects of cleavage fracture in steel. Metallurgical Transactions A 1983;14:1349 55.

[7] Beremin FM. A local criterion for cleavage fracture of a nuclear pressure vessel steel. Metallurgical Transactions A 1983;14:2277 87.

[8] Neal D, Vangel M. Statistical analysis of mechanical properties. In: ASM Handbook Committee (Ed.), Composites, engineered materials handbook. ASM International, vol. 1, 1987. p. 3027.

[9] McCool JT. Inference on Weibull percentiles and shape parameter from maximum likelihood estimated. IEEE Transactions on Reliability $1970 ; \mathrm{R} 19: 29$

[10] Bergman B. Estimation of Weibull parameters using a weight function. Journal of Materials Science Letters 1986;5:611 4.

[11] Faucher B, Tyson R. On the determination of Weibull parameters. Journal of Materials Science Letters 1988;7:1199 203.

[12] Thoman DR, Bain LJ, Antle CE. Inferences on the parameters of the Weibull distribution. Technometrics 1969;11(3):445 61.

[13] Fernández-Sáez J, Chao J, Durán J, Amo JM. Estimating lower-bound fracture parameters for brittle materials. Journal of Materials Science Letters 1993;12:1493 6 .

[14] Barbero E, Fernández-Sáez J, Navarro C. On the estimation of percentiles of the Weibull distribution. Journal of Materials Science Letters 1999;18(17):1441 3.

[15] Khalili A, Kromp K. Statistical properties of Weibull estimators. Journal of Materials Science 1991;26:6741 52.

[16] Barbero E. Desarrollo y caracterización mecánica de nuevos materiales compuestos de matriz cerámica y refuerzo cerámico. $\mathrm{PhD}$ thesis, Carlos III University of Madrid, Madrid, 1999 (in Spanish). 